\title{
FUNDAÇÃO OSWALDO CRUZ experiência centenária em biologia e saúde pública
}

\author{
Paulo Marchiori Buss
}

Paulo Gadelha

\begin{abstract}
Resumo: O artigo descreve a experiência da Fiocruz no campo da ciência e tecnologia em saúde, ressaltando seu modelo de planejamento e gestão, bem como destacando as principais características dos programas institucionais de pesquisa e desenvolvimento tecnológico; ensino; produção; serviços de referência e assistência; informação e comunicação; e desenvolvimento institucional. Palavras-chave: instituições científicas; Fiocruz; pesquisa e desenvolvimento tecnológico.
\end{abstract}

Abstract: This article describes the role of Fiocruz in the area of health-related science and technology, focusing in particular on its planning and management model, as well as the main principles of its institutional programs in the areas of technological research and development, teaching, production, referral and assistance services, information and communication, and institutional development.

Key words: scientific institutions; Fiocruz; technological research and development.

\footnotetext{
Gerar, absorver e difundir conhecimentos cientificos e tecnológicos em saúde, pelo desenvolvimento integrado de atividades de pesquisa, ensino, informação, tecnologia e produção de bens e serviços, com a finalidade de proporcionar apoio estratégico ao Sistema Único de Saúde e contribuir para a melhoria da qualidade de vida da população $e$ para o exercício da cidadania.
}

Missão da Fiocruz/ III Congresso Interno

$\mathrm{A}$ Fundação Oswaldo Cruz (Fiocruz) surgiu em 1900 com a denominação de Instituto Soroterápico Federal como resposta a uma crise sanitária representada pela ameaça de extensão da epidemia de peste bubônica do porto de Santos para o Rio de Janeiro, então capital do país. Sua finalidade básica consistia na substituição de produtos importados e aprimoramento tecnológico de soros e vacinas (Benchimol, 1989). Esse projeto foi logo ampliado pela ação de Oswaldo Cruz, articulada ao processo de construção da nacionalidade do país. O saneamento e a reurbanização da capital, em que se articulava a lógica econômica centrada na intermediação financeira da exportação de produtos primários, formavam o projeto central dos primórdios da República. Tratava-se, para além da li- beração dos portos, de criar um novo símbolo para a sede da República, superando a imagem de cidade colonial por outra, inspirada nas modernas metrópoles européias. Para tanto, a nova higiene, surgida com a revolução pasteuriana, desempenhava papel central.

Inspirado no Instituto Pasteur, o novo instituto constituiu-se fora da universidade e com um modelo organizacional que reunia as atividades de pesquisa, ensino, produção e prestação de serviços. Ao contrário da natureza privada de sua contraparte francesa, vinculou-se aos órgãos federais de saúde pública, o que lhe conferiu importante papel na formulação de políticas e ações de saúde pública. Por sua vez, a arrecadação de recursos com a venda de produtos e a excelência científica conferiram uma "autonomia relativa" diante de instâncias centrais. Sua história centenária revela um expressivo sucesso, tornando-se referência internacional em sua área de atuação e um símbolo estreitamente associado à construção de nacionalidade e cidadania do Brasil. ${ }^{1}$

Foi fundamental para essa performance a forte interação com a dinâmica social e política que marca sua origem, sendo exemplo recente o papel que vem desempenhando no projeto da reforma sanitária. Outras características 
vêm sendo apontadas, à semelhança da análise realizada por De Masi (1997) referente ao Instituto Pasteur: "passagem da little science para big science, reunindo atividades de inovação e coordenação; pesquisa multidisciplinar; importância conferida à difusão do conhecimento; superação da dicotomia "ciência pura" e "ciência aplicada"; internacionalismo; mobilidade (missões, filiais, etc.); valorização da estética e sinergia entre tradição e inovação".

Esses traços retrospectivos adquirem relevância quando se enfrenta um contexto de mudanças significativas na dinâmica de produção da ciência e tecnologia e, em especial, das Instituições Públicas de Pesquisa (IPP), marcadas por rupturas de paradigmas técnico-científicos, transformações do papel do Estado, novos padrões concorrenciais e globalizações dos mercados. Constata-se, ainda, em âmbito mundial, uma tendência ao aumento de custos da atividade de pesquisa com redução de orçamentos, associados a rigidez institucional e deficiências na mudança de ambiente necessário em face dos novos desafios da globalização e competitividade por recursos (Salles Filho, 2000). Esse quadro torna-se particularmente relevante no contexto nacional, no qual, ao contrário de outros países, as Universidades e IPPs desempenham papel central no desenvolvimento da ciência e da tecnologia, ao mesmo tempo em que o caráter público dessas instituições vem sendo questionado por discursos de moldes neoliberais.

Salles Filho sumariza a tendência internacional das funções públicas das IPPs: geração de conhecimentos estratégicos; formulação de políticas públicas; execução de políticas públicas; geração de oportunidades de desenvolvimento econômico, social e ambiental; arbitragem. A Fiocruz participa em todos esses aspectos, seja por sua ação direta, seja por seu papel de suporte científico-tecnológico a outras agências do Ministério da Saúde (MS). O sucesso na execução dessas funções das IPPs seria dado por sua competência em: ser reconhecida como instituição de excelência; ser identificada pelo setor produtivo como instituição capaz de resolver problemas; visibilidade social, modernização da produção, sustentabilidade ambiental, etc.; fazer-se presente enquanto instituição formuladora/executora; e ser eficaz em public accounting (Salles Filho, 2000).

Para as IPPs e, em especial, a Fiocruz, torna-se, portanto, cada vez mais relevante consolidar sua legitimidade lastreada na adequação de suas atividades às demandas sociais. A reforma do Estado, com o reforço de seu papel de regulação e redistribuição social - o que implicou na reconformação do sistema de saúde, com a delegação de responsabilidades a Estados e Municípios - e as mudanças nos quadros sociodemográfico e sanitário do país, associados à implementação por parte do MS de políticas e programas identificados com reivindicações históricas do movimento sanitário na área de medicamentos, saúde da família, regulação da assistência complementar e de fomento para a ciência e tecnologia em saúde, colocam desafios e oportunidades para o fortalecimento do caráter público e estatal da Fiocruz e de suas funções estratégicas na tomada de decisões governamentais.

Por sua vez, as transformações no campo da ciência e tecnologia, em especial nas áreas de biologia molecular e biotecnologia, exigem um esforço a longo prazo para manter sua capacidade de dar respostas de forma competitiva nacional e internacionalmente.

O debate nacional em torno desse contexto teve na Conferência Nacional de Ciência, Tecnologia e Inovação, realizada em novembro pelo Ministério da Ciência e Tecnologia (MCT, 2001), seu momento maior. Com a premissa de que a afirmação do país na economia globalizada requer o incremento da produção de bens de valor tecnológico agregado e partindo do diagnóstico do descompasso nacional entre uma significativa produção de conhecimentos e qualificação de pessoal, seus efeitos sobre o setor produtivo e a resolução de problemas sociais, o tema da inovação foi eleito como eixo central para a consolidação de novas formas de produzir, aplicar e valorizar o conhecimento por meio de novos agentes e arranjos (redes, cadeias produtivas, clusters, etc.) Isso traz implicações sobre o papel do Estado, formas de financiamento e reorganização das IPPs, com ênfase na atividade de planejamento e gestão de CT\&I (MCT, 2001). Essa mudança de mentalidade é fundamental para consolidar a pertinência da atividade científica $v i s-a ̀-v i s$ ao projeto nacional voltado para o desenvolvimento econômico e social e a Fiocruz vem direcionando seus esforços nesse sentido, e a questão da inovação é um dos eixos que presidem seu Plano Quadrienal 2001-2005 (Fiocruz, 2001a).

O projeto de Lei de Inovação, apresentado durante a CNCT\&I, é um dos instrumentos centrais desse objetivo, juntamente com a constituição dos fundos setoriais e a internalização de iniciativas de planejamento, inspiradas em experiências internacionais que buscam definir prioridade em C\&T com base no aperfeiçoamento de métodos de planejamento e avaliação de tecnologias-chave, a exemplo, no caso brasileiro, da constituição do Centro de Estudos Estratégicos de CT\&I, de projetos como o Prospectar, no marco de referência do Livro Verde de C\&T e da Agenda de C\&T do MS (MS, 2001). 
É neste contexto que se reorganiza a Fundação Oswaldo Cruz, cujas principais características são apresentadas a seguir.

\section{MODELO DE PLANEJAMENTO E GESTÃO DA FIOCRUZ}

\section{A Fiocruz no Contexto da Saúde e da Ciência e Tecnologia}

Por sua localização institucional histórica (origem e parte integrante do Ministério da Saúde), por sua tradição de instituição centralmente voltada para a produção de conhecimentos e pelo objeto central de seu trabalho técnico-científico (a saúde e seus determinantes biológicos e sociais), a Fiocruz articula - não sem as dificuldades inerentes a esta posição - os setores de saúde e de ciência e tecnologia. Tem sido, ao longo de seus mais de 100 anos de existência, uma das principais instituições científicas do país (sejam quais forem os indicadores utilizados) e, ao mesmo tempo, um dos mais importantes suportes técnico-científicos às ações de saúde pública empreendidas pelo Ministério da Saúde.

Portanto, é neste espaço caracterizado como C\&T em Saúde ${ }^{2}$ que pesquisa, ensina e produz a Fundação Oswaldo Cruz.
A base primordial do trabalho institucional foi e continuará sendo sempre a pesquisa em saúde, como fonte do conhecimento da realidade e das alternativas para enfrentála. Define-se pesquisa em saúde no contexto da Fiocruz (Buss, 2001) como "uma pesquisa que combina harmonicamente os conhecimentos sobre os mecanismos íntimos das nossas principais doenças - propiciados pela pesquisa biomédica e biológica, a medicina e a biologia experimentais, fonte de grande prestígio para Manguinhos e para o país - com a pesquisa clínica, que identifica a expressão do processo de adoecimento nos indivíduos, bem como suas soluções, com a pesquisa em saúde pública, isto é, as pesquisas epidemiológica, social e histórica, assim como a investigação sobre políticas, sistemas e serviços de saúde, que agregam estratégias fundamentais para um enfrentamento global dos problemas de saúde".

A Constituição Federal de 1988 criou o Sistema Único de Saúde e definiu, entre suas atribuições, "incrementar, em sua área de atuação, o desenvolvimento científico e tecnológico". De outro lado, a Lei no 8.080, de 19/09/1990, dispõe sobre os diversos campos que compõem o SUS. Simplificadamente, pode-se dizer que o SUS possui dois grandes campos de atuação, o da saúde individual (preventiva e curativa) e o da saúde coletiva ou populacional (saúde pública).

DIAGRAMA 1

Situação da Fiocruz no contexto do Sistema Único de Saúde

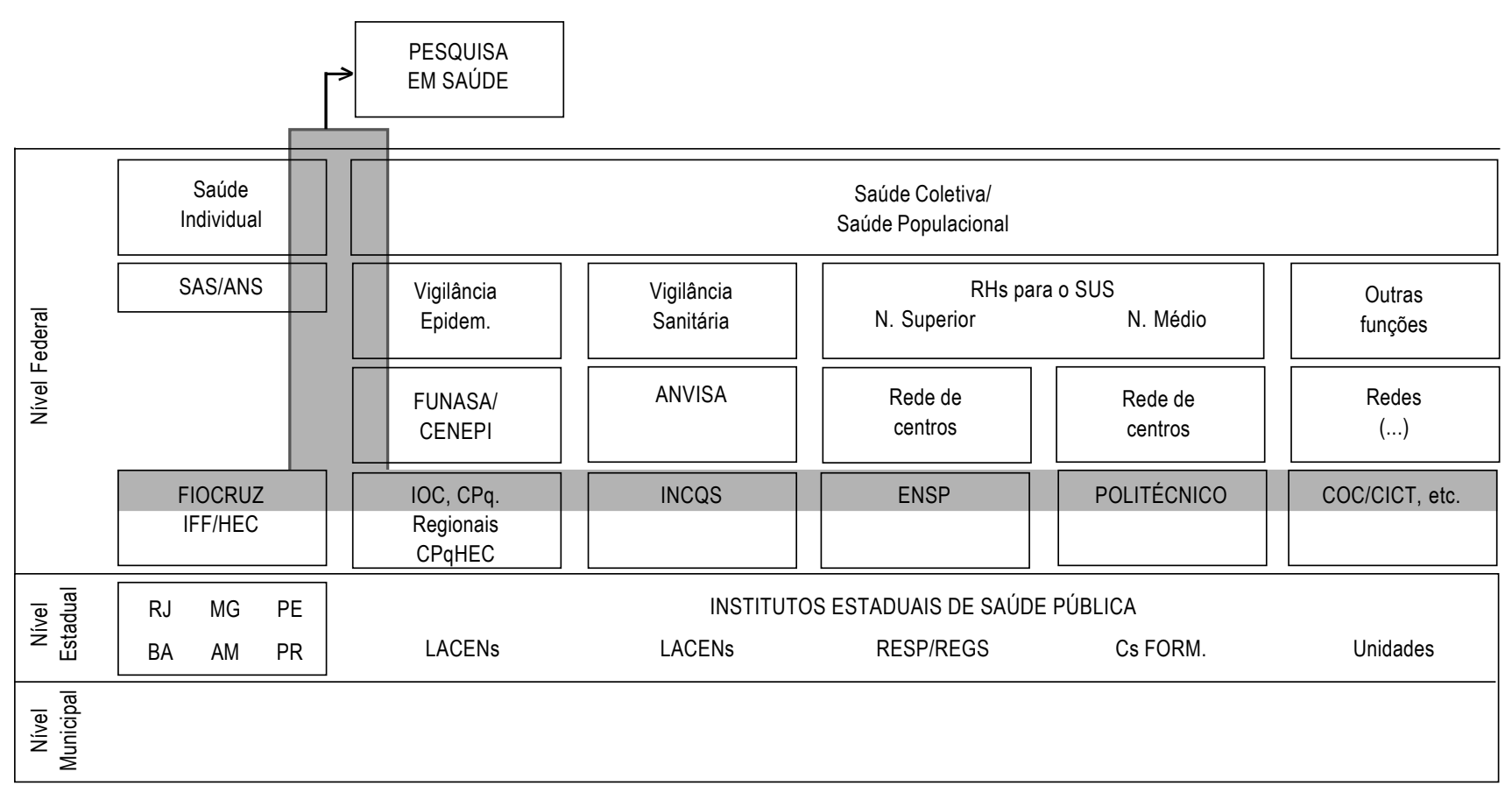


No Diagrama 1, procura-se caracterizar a posição da Fiocruz, como órgão de C\&T em saúde e, simultaneamente, integrante do nível federal do Sistema Único de Saúde em relação aos dois grandes campos de atuação do SUS.

Todo o planejamento institucional procura atender a estas duas vocações institucionais mediante cinco programas finalísticos (pesquisa e desenvolvimento; ensino; produção; serviços de referência; informação e comunicação) e um programa de desenvolvimento institucional e gestão. No início da gestão atual (2001-2004) foi estabelecido um Plano Quadrienal 2001-2005 (Fiocruz, 2001a), cobrindo com objetivos e metas os seis programas, que foram aprovados nas diversas instâncias de decisão da Fiocruz (ver a seguir), ao que se seguem Planos (anuais) de Objetivos e Metas - os POMs, globais e por Unidade Técnica.

A seguir, são apresentadas as instâncias e os mecanismos decisórios, bem como as principais características programáticas para o quadriênio.

\section{Instâncias e Mecanismos de Decisão}

Congresso Interno - O modelo de gestão da Fiocruz constituiu-se tendo como premissa central a importância de mecanismos participativos na elaboração de seu planejamento estratégico e implementação de políticas e programas. Em 1987, durante a gestão de Sérgio Arouca, criouse uma experiência inédita no âmbito da administração pública, o Congresso Interno, depois incorporado ao novo Estatuto da Fiocruz como órgão máximo de representação, com competência para deliberar, no âmbito interno da Instituição, sobre assuntos estratégicos referentes ao projeto institucional e alterações do regimento interno e estatuto.

Composto de delegados eleitos em todas as Unidades, conforme princípio de representações mínima, média e máxima de acordo com o número de funcionários, o Congresso Interno acontece no início de cada mandato presidencial (de 4 anos) da Fiocruz, com o objetivo de atualizar diretrizes do projeto institucional e pactuar os termos de compromisso entre gestores e o conjunto da comunidade.

Sua preparação envolve a realização de seminários abertos à participação de toda a comunidade, que contribui, juntamente com convidados externos, para a fundamentação teórica e a avaliação de cenários e conclui-se com a plenária deliberativa composta pelos delegados eleitos.

A experiência do Congresso Interno é extraordinária. Pesquisadores, tecnólogos, técnicos, administradores, enfim, todo espectro da comunidade da Fiocruz realiza um tour de force de reflexão e deliberações de grande densidade e conseqüências para o futuro institucional. A adesão a esse projeto, decorrência do processo participativo de sua construção, tem garantido a capacidade de implementar programas e superar diversidades.

$\mathrm{O} 1{ }^{\circ}$ Congresso Interno, verdadeira "estatuinte" do período de redemocratização, marcou o nascimento das bases do projeto institucional e do atual modelo de gestão. Foram definidos os fundamentos da identidade institucional e a abrangência de suas áreas de atuação, as visões que norteiam os macroprocessos de pesquisa, desenvolvimento tecnológico, produção, ensino, serviços e informação e comunicação e definidas prioridades programáticas. Desenhou-se o modelo de gestão e foram instituídos, além do Congresso Interno já mencionado, os Conselhos Deliberativos da Fiocruz - constituídos pela presidência, diretores de unidades e representantes da associação de servidores - e das Unidades Técnico-Científicas, as Câmaras Técnicas - órgãos de assessoramento da presidência e $\mathrm{CD} /$ Fiocruz - e o mecanismo eleitoral como forma de indicação, em listas tríplices, do Presidente da Fiocruz e dos diretores das Unidades Técnico-Científicas.

A seqüência dos Congressos Internos, hoje em sua quarta versão, e várias plenárias extraordinárias, retrata a atualização das agendas nacionais no campo da ciência e tecnologia em saúde e sua tradução no desenvolvimento institucional da Fiocruz. O $2^{\circ}$ Congresso Interno tratou centralmente da relevância pública, controle social e incremento da eficiência, da qualidade e flexibilidade das ações desenvolvidas e elaborou os fundamentos que definiram a posição da Fiocruz perante diretrizes da Reforma do Estado, com a reafirmação do caráter estratégico, público e estatal da Fiocruz e a adoção de um modelo de gestão de resultados, implementado mediante termos de compromissos internos e externos. $\mathrm{O} 3^{\circ}$ Congresso Interno deu novos passos nesse sentido, qualificando processos para consolidar o papel estratégico da Fiocruz, consolidar mecanismos de gestão e pleitear a qualificação enquanto Agência Executiva. Esses grandes temas traduzem-se em análises e propostas relativas aos macroprocessos em que a instituição está envolvida.

Conselho Deliberativo - O Conselho Deliberativo da Instituição está formado pelos Diretores das Unidades Técnico-Científicas (UTC) e Técnicas de Apoio (UTA), em número de 17, além de 1 (um) representante dos trabalhadores da Instituição. Sua função é detalhar e operacionalizar as macropolíticas definidas no Congresso Interno, também discutindo e aprovando os programas e respectivos orçamentos anuais e plurianuais da Instituição. 
Além dessas funções mais ligadas ao cotidiano institucional, o CD/Fiocruz tem desempenhado um papel político muito importante de mediador das crises institucionais que eventualmente acometem a Instituição. Um dos exemplos marcantes dessa atuação ocorreu no Governo Collor, quando o Ministério da Saúde questionou a legitimidade da elaboração de lista tríplice para escolha do Presidente da Fiocruz, impasse que foi adequadamente contornado pelo $\mathrm{CD} /$ Fiocruz.

Câmaras Técnicas - O Conselho Deliberativo (assim como a Presidência) da Fiocruz é apoiado, tecnicamente, quanto aos programas finalísticos da Instituição (pesquisa e desenvolvimento tecnológico; ensino; assistência e serviços de referência; produção; informação e comunicação; e gestão) pelas Câmaras Técnicas, organizadas com representantes de suas UTC e UTA e presididas pelos respectivos vice-presidentes setoriais. Sua função principal é propor arranjos programáticos e procedimentos técnicos e gerenciais ao $\mathrm{CD} /$ Fiocruz para a implementação dos programas finalísticos da Instituição.

Coletivo de Dirigentes - O Coletivo de Dirigentes da Fundação Oswaldo Cruz é o primeiro escalão ampliado da Instituição. Foi implantado na atual gestão, em outubro de 2001. Reúne cerca de 130 dos principais dirigentes da Fundação, incluindo os membros do CD/Fiocruz (Diretores das Unidades), vices-presidentes, diretores e chefes de departamentos da administração central (Dirad, Dirac, Direh, Procuradoria, Auditoria e Assessorias da Presidência) e chefes de Departamentos ou equivalentes de todas as Unidades Técnicas da Fiocruz.

A proposta de criá-lo e reuni-lo periodicamente acompanha a tendência de inúmeras organizações públicas e privadas de todo o mundo, que reconhecem a necessidade de "capilarizar" as decisões políticas tomadas até os escalões técnicos responsáveis pela implementação de tais decisões, "onde as coisas realmente acontecem". Além disso, situando a Instituição e tais políticas no macroambiente socioeconômico, político e científico em que se encontram imersas.

A Fiocruz possui uma longa e vitoriosa tradição de participação na formulação de políticas, por meio do Congresso Interno e do CD/Fiocruz, antes referidos. O Coletivo de Dirigentes vem preencher uma lacuna de caráter mais operacional.

Entre seus objetivos encontra-se o de propiciar o conhecimento mútuo e o intercâmbio de idéias entre principais dirigentes da Instituição, "pessoalizando" mais as relações entre eles, diminuindo a "atomização" insitucional e criando oportunidades de trabalhos conjuntos; conhecer e debater as oportunidades e dificuldades das conjunturas socioeconômicas, políticas e científicas para a Instituição; conhecer e sugerir aperfeiçoamentos às macropolíticas da Instituição, entre as quais, neste momento da gestão 2001-2004, estão as "Diretrizes para a Formulação do Plano Quadrienal 20012005"; conhecer e sugerir aperfeiçoamentos aos mecanismos de implementação, monitoramento e avaliação das ações decorrentes do Plano Quadrienal, etc.

\section{PROGRAMAS INSTITUCIONAIS}

\section{Pesquisa e Desenvolvimento Tecnológico em Saúde}

Desde seus primórdios, os pesquisadores da Fiocruz têm buscado articular a geração de novos conhecimentos científicos com sua aplicação. Esta não é uma tarefa fácil, nem linear. No entanto, num mundo científico competitivo e num estágio de desenvolvimento social brasileiro que cada vez mais exige respostas efetivas das Instituições científicas, particularmente as públicas e estatais, a Fiocruz coloca o fortalecimento da função desenvolvimento tecnológico como perspectiva prioritária para o futuro.

Isto não implica que o desenvolvimento científico será descuidado na Instituição, fonte de grande prestígio para Manguinhos e para o Brasil. Ao mesmo tempo em que a Instituição prima pelo desenvolvimento tecnológico e criam-se mecanismos para materializar essa macroorientação, a perspectiva é também manter uma adequada gestão da diversidade para o desenvolvimento científico, como se verá a seguir, que é também uma das perspectivas para o futuro da Instituição.

O Livro Verde de Ciência e Tecnologia (MCT, 2001), preparado como subsídio à Conferência Nacional de Ciência, Tecnologia e Inovação, recentemente realizada em Brasília/DF, diagnostica o sistema de C\&T em saúde como fragmentado e ineficiente, caracterizando-se pela fragilidade do planejamento, pela ausência de mecanismos para o estabelecimento de prioridades e por um sistema de informações precário. A conseqüência tem sido a duplicação de esforços, a desarticulação das iniciativas em andamento e a baixa aplicabilidade dos esforços de pesquisa e desenvolvimento (P\&D) no âmbito do Sistema Único de Saúde (SUS) (grifo nosso).

Aponta critérios para a definição de prioridades, a saber: riscos das patologias para a saúde pública; geração 
de conhecimentos estratégicos; patologias específicas à situação brasileira; competência científica existente e potencial de irradiação dos grupos de excelência; e aplicabilidade dos resultados aos problemas prioritários da agenda da saúde pública.

No entanto, o documento do CD/Fiocruz sobre diretrizes para a formulação do Plano Quadrienal (Fiocruz, 2001a) recomenda "o estabelecimento de mecanismos permanentes de análise de demandas de C\&T em saúde, que considerem os contextos sociosanitário e científico-tecnológico e que permitam o estabelecimento mais consensual de prioridades institucionais". E define ainda duas medidas:

- Criar o Programa de Desenvolvimento Tecnológico de Insumos em Saúde (PDTIS), (Fiocruz, 2002a), visando ao desenvolvimento de novas vacinas, reagentes para diagnóstico, fármacos e medicamentos e produtos para o controle de vetores, bem como o aperfeiçoamento de produtos existentes; e

- Criar o Programa de Desenvolvimento Tecnológico em Saúde Pública (PDTSP), (Fiocruz, 2002b), visando ao desenvolvimento de metodologias e tecnologias em saúde populacional e individual.

O foco em insumos em saúde, vale dizer, vacinas, kits e reativos diagnósticos, produtos para controle de vetores, fármacos e medicamentos justifica-se pela grande experiência institucional nestas áreas; pela importância desses insumos para a saúde pública - seja pelo alto valor econômico agregado de tais produtos, seja pela melhora na qualidade assistencial agregada por eles ao sistema de saúde; e pela relevância e pelos desafios científicos que tais temas colocam para os pesquisadores da Instituição.

O foco em saúde pública tem duas dimensões: saúde populacional e saúde individual. Reúne-se aqui a pesquisa e desenvolvimento em sistemas e serviços de saúde, a pesquisa epidemiológica e a pesquisa clínica. Justificase também pela considerável experiência acumulada na Instituição nestas áreas, assim como pela qualidade que agregam a tomada de decisão na gestão dos sistemas, serviços e programas de saúde, como no manejo clínico de problemas de saúde individuais.

Tais Programas de Desenvolvimento Tecnológico (PDTs) foram montados em 2001 e estão sendo implementados em 2002 e anos subseqüentes; são financiandos por fundos específicos criados e gerenciados pela Presidência e tem os mecanismos de seleção de projetos baseados na qualidade científica e na relevância para o sis- tema de saúde. O PDTIS recebe recursos oriundos dos resultados financeiros da produção de insumos na Fiocruz; o PDTSP, das atividades assistenciais e de consultoria, assim como recursos do Tesouro, já que por si só não tem capacidade de gerar a massa de recursos necessária. Os critérios para a seleção de projetos são amplamente discutidos pelas várias instâncias envolvidas (Câmara Técnica de Pesquisa e Desenvolvimento Tecnológico; $\mathrm{CD} /$ Fiocruz e outras instâncias adequadas) e incluem qualidade técnico-científica interna, relevância social e clareza nos resultados a serem alcançados e sua utilização.

Gestão da Diversidade - Esses dois programas indutivos de apoio a P\&D são complementados pelo Programa de Apoio à Pesquisa em Saúde (Papes), que financia, com recursos próprios da Fiocruz alocados também em um fundo específico, a pesquisa científica desenvolvida em seus vários Institutos. O principal critério para a seleção dos projetos apresentados é sua qualidade científica, aferida por bancas externas especialmente convidadas para este fim. Como são, em geral, recursos de pequena monta (entre R\$ 10 e R \$ 40 mil - valores de 2002), funcionam como seed money e estimulam o pesquisador a buscar recursos que os complementem nos processos competitivos externos.

Uma excelente oportunidade para aferir a qualidade dos projetos de pesquisa da Fiocruz e para a divulgação e coletivização do conhecimento produzido é a Bienal da Pesquisa, evento que reúne bi-anualmente trabalhos desenvolvidos no âmbito da maioria dos projetos de pesquisa em desenvolvimento. Em 2001, a Fiocruz tinha cerca de 1.250 projetos de pesquisa em desenvolvimento e produziu mais de 1.000 artigos e capítulos de livro, além de terem seus pesquisadores participado com trabalhos em mais de 850 congressos e outros eventos científicos.

As Comissões de Ética na Pesquisa e no Uso de Animais de Laboratório estabelecem regras e examinam sua rigorosa aplicação nos projetos desenvolvidos no âmbito da Instituição.

Apoiar o desenvolvimento científico por meio da disponibilização de insumos e infra-estrutura básicos via orçamento regular das Unidades, reforçados pela constituição de fundos institucionais para o desenvolvimento de projetos, conforme pauta de prioridades. Assim se entende a estratégia de implementação da "gestão da diversidade", isto é, apoiar o potencial criativo dos pesquisadores da Instituição. 
Formulação de Prioridades em P\&D - A formulação de prioridades em $P \& D$ na Fiocruz vem sendo guiada pelas agendas governamentais de saúde e ciência e tecnologia, para o que estão sendo otimizadas as competências específicas da instituição e as tendências do desenvolvimento científico e tecnológico, segundo diretrizes estabelecidas pelos fóruns institucionais, com utilização de mecanismos e instrumentos de gestão de C\&T adequados aos fins pretendidos.

Já se afirmou, neste artigo, que o próprio Livro Verde aponta critérios para a definição de prioridades na área de C\&T em saúde, a saber: riscos das patologias para a saúde pública; geração de conhecimentos estratégicos; patologias específicas à situação brasileira; competência científica existente e potencial de irradiação dos grupos de excelência; e aplicabilidade dos resultados aos problemas prioritários da agenda da saúde pública.

Tomando como referência a necessidade de articulação entre geração de conhecimentos e as necessidades do sistema de saúde, são consideradas tanto a situação sanitária vigente quanto a situação na área de pesquisa e desenvolvimento em saúde, mesmo que em traços muito genéricos, para a definição de alguns princípios norteadores da visão de política de CT\&I em saúde na Instituição.

No que concerne ao quadro sanitário vigente, ainda que vários indicadores de saúde tenham melhorado nos últimos anos, como a mortalidade infantil e o controle das doenças imunopreveníveis, persistem importantes enfermidades infecciosas e parasitárias como as diarréias, infecções respiratórias agudas, febre amarela, malária, entre muitas outras, determinando altas taxas de morbidade e mortalidade. O aumento das chamadas doenças emergentes e reemergentes, como Aids/HIV, dengue, resistência bacteriana, hantavirose, arenavírus e outras arboviroses, são preocupantes. As viroses oncogênicas e as hepatites virais compõem, finalmente, o expressivo elenco das doenças infecto-parasitárias, com que historicamente a Fiocruz se enfrenta.

Além disso, verifica-se o rápido aumento de moléstias típicas dos países desenvolvidos, como as doenças cardiovasculares e as neoplasias. A migração das populações de regiões mais pobres para os grandes centros urbanos, por sua vez, com um crescimento desordenado e grande contingente populacional vivendo nas periferias, sem infraestrutura mínima de saneamento e habitação, vem resultando em aumento substantivo da demanda ao sistema de saúde. Outros problemas também afetam diretamente a saúde e a doença, como as deficiências de saneamento básico, as carências nutricionais, a poluição ambiental, o estresse, as doenças alérgicas e a violência urbana.

A diversidade e a complexidade dos problemas existentes, característicos do quadro de polarização epidemiológica vigente, tornam ainda mais importante que sejam definidas estratégias e prioridades adequadas de CT\&I. É neste contexto maior da saúde, que remete para o tema da qualidade de vida e do bem-estar, que devem ser colocados os desafios estratégicos da CT\&I na área.

O documento preparado pela Fiocruz para a Conferência Nacional de Ciência, Tecnologia e Inovação (Fiocruz, 2001b) considera que comissões ad hoc de especialistas e representantes da sociedade civil seriam instrumentos essenciais para o detalhamento de prioridades no interior de cada grande área do campo da saúde. Não se furtou, entretanto, de listar as grandes áreas que deveriam estar englobadas no campo da CT\&I em saúde, no interior das quais haveria a necessidade de estabelecimento de programas e projetos prioritários, seguindo critérios que também apontam no corpo do documento.

\section{Ensino na Fiocruz e seu Papel Estratégico no SUS}

Desde seus primórdios, como também nos dias atuais, a pesquisa em saúde de alta qualidade tem sido a base principal dos programas de ensino da Fiocruz. Eles se desenvolvem nas áreas biológicas/biomédicas, de saúde pública, saúde materno-infantil, história das ciências e da saúde e do controle de qualidade em saúde (Fiocruz, 2002c) e encontram-se entre os melhores do país no processo de avaliação do sistema Capes/MEC.

Além dos Mestrados e Doutorados em desenvolvimento, os cursos da Fiocruz abrangem ainda o nível médio e as categorias que integram o lato sensu, apresentando uma multiplicidade de áreas temáticas de afinidade com as linhas de pesquisa e com as demandas de formação dos profissionais de saúde para os Sistemas de Saúde e de C\&T em Saúde (Fiocruz, 2002c).

Nos anos recentes, estruturaram-se iniciativas inovadoras como o Programa de Educação a Distância (Proead); o Programa de Apoio ao Ensino Técnico com ênfase na formação de professores e foco na relação ensino - pesquisa (Paetec); os Mestrados Interinstitucionais; o Sistema de Acreditação Pedagógica; o Projeto Escola de Governo em Saúde (estratégia estruturante da relação do ensino da Fiocruz com o Sistema de Saúde) e o Programa de Educação para a Ciência em suas múltiplas dimensões. 
Em 2001/2002 estão sendo implantados Mestrados Profissionais em diversas áreas, incluindo o Mestrado Profissional em C\&T em Saúde. Este se dedica prioritariamente à formação de quadros e ao desenvolvimento de metodologias e tecnologias de gestão em C\&T próprias da instituição, podendo ampliar-se no futuro para outros órgãos congêneres.

Para a reorientação do ensino na Fiocruz busca-se sinergicamente a articulação com atores representativos das demandas da sociedade; a integração entre os programas e projetos de ensino; a modernização de sua gestão; e a valorização do vínculo entre ensino e pesquisa, bem como das experiências bem-sucedidas.

Pós-Graduação Sensu Stricto - Em 2001 estavam em desenvolvimento na Instituição oito programas de pós-graduação, com 898 mestrandos e doutorandos matriculados, no interior dos quais foram defendidas 154 dissertações de mestrado e 66 teses de doutorado.

Os mestrandos e doutorandos da Fiocruz vinculam-se estreitamente às linhas de pesquisa em desenvolvimento na Instituição, representando, por isto mesmo, segmento importante na produção de conhecimentos e inovações. Toda a produção científica da pós-graduação da Fiocruz é coletivizada anualmente pela já tradicional Jornada Científica da Pós-Graduação, que se realiza simultaneamente à Reunião Anual da Iniciação Científica, que coletiviza a produção dos alunos desta modalidade de treinamento profissional.

Escola de Governo em Saúde e Mestrados Profissionalizantes - A Escola de Governo em Saúde é uma reorientação estratégica dos programas de ensino, pesquisa e cooperação técnica da Fiocruz, que visa:

- Preparar quadros dirigentes capacitados para a gestão estratégica e operacional de sistemas, serviços, programas e organizações de saúde;

- Produzir conhecimento adequado e passível de ser imediatamente aplicado no desenvolvimento do sistema de saúde, traduzido em metodologias e tecnologias assistenciais e gerenciais;

- Cooperar diretamente com órgãos gestores e unidades operacionais do Sistema Único de Saúde, nas três esferas governamentais, na solução de problemas concretos de gestão;

- Fomentar e articular outras instituições de ensino e pesquisa em Saúde Coletiva com vista a construir rede nacional de escolas de governo em saúde.
Foi implantada inicialmente na ENSP, a partir de 1998, visando estruturar a formação e educação permanente de gestores públicos de sistemas, serviços, organizações e programas, bem como cooperar na implantação de abordagens inovativas na assistência médica, na saúde pública e na formulação e avaliação das políticas de saúde.

O desafio central do programa é construir um perfil profissional estratégico, que talvez possa ser chamado de "gestor público da saúde", a reunir competências do novo profissional de saúde pública (capacidade de processar e enfrentar problemas sanitários em sua complexidade técnica e social), com o moderno gestor de sistemas e serviços de saúde (capacidade de gerenciamento técnico e negociação política).

A partir de 2002 tal estratégia vem sendo estendida às demais Unidades da Fiocruz, visando à capacitação de gestores de diversos programas do SUS, que incluem a vigilância em saúde (vigilâncias sanitária e epidemiológica), laboratórios de saúde pública e a área de informação e comunicação em saúde, entre outras.

Os Mestrados Profissionais são programas de ponta nas linhas de trabalho da Escola de Governo em Saúde no âmbito da Fiocruz. Eles também se iniciam na ENSP, mas estão em processo de implantação nas áreas antes mencionadas, muitas das quais localizadas em outras Unidades da Instituição.

Em futuro próximo, após a consolidação dos programas presenciais, os Mestrados Profissionais também deverão ser oferecidos pelo Programa de Educação a Distância da Fiocruz, que já oferece cursos de especialização e outros, como se apresenta a seguir.

Educação a Distância - Desde 1998 a Fiocruz desenvolve, pela Escola Nacional de Saúde Pública, um programa de educação a distância.

As novas tecnologias de informação e comunicação, pela sua virtualidade de expandir a amplitude e a velocidade de circulação de conhecimentos, apresentam-se como naturalmente capazes de viabilizar as exigências de escala e custo-efetividade (maior eficiência na utilização de recursos educacionais - seja como capacidade institucional instalada, seja como recursos de custeio) desses processos educacionais. Some-se a isso a possibilidade de viabilizar processos educacionais sem que o profissional se afaste de seu local de trabalho, o que é de altíssima relevância para a clientela da saúde pública.

A EAD da Fiocruz tem hoje cerca de 6 mil alunos regularmente matriculados, em cursos de especialização e 
aperfeiçoamento. Neste total não estão computados os alunos de projetos especiais da EAD, como o de formação de multiplicadores para o Programa de Formação de Auxiliares de Enfermagem (Profae) do Ministério da Saúde, que somam cerca de 9 mil enfermeiras matriculadas; a formação de cerca de 43 mil conselheiros de saúde e 2,5 mil juízes e procuradores do Ministério Público; e o curso para formar 20 mil agentes de vigilância em saúde, numa iniciativa da Fiocruz, por intermédio da Escola Politécnica da Saúde e da Funasa, órgão do Ministério da Saúde. Tais números gigantescos apenas reforçam o sentido e o significado da EAD na área da saúde, num país das dimensões como o Brasil.

Outros dois programas de ensino de grande importância estão em desenvolvimento na Instituição: o Programa de Vocação Científica (Provoc), destinado a alunos de nível médio de escolas selecionadas do Rio de Janeiro; o Programa de Iniciação Científica, para alunos dos últimos períodos de cursos universitários, que são fonte fundamental para os programas de mestrado e doutorado.

\section{Produção}

Resultante do desenvolvimento de produtos com base na pesquisa própria e de acordos de transferência de tecnologia, a Fiocruz apresenta importante programa de produção de bens (insumos) para o setor saúde, traduzido em medicamentos, vacinas e kits diagnósticos para os principais programas de saúde pública do país.

Duas Unidades de desenvolvimento tecnológico e produção fazem parte da estrutura da Fiocruz: Bio-Manguinhos, produtor de vacinas e kits diagnósticos; e FarManguinhos, que produz medicamentos.

Em 2001, a Fiocruz produziu cerca de 100 milhões de doses de vacina (o que representa mais de $40 \%$ do que se consome no país), dos seguintes produtos: vacinas antipólio, anti-sarampo, contra o Hemophilus influenza $B$, tetra-valente (DTP+HiB), anti-meningocócica e contra a febre amarela (da qual é o mais importante produtor mundial). Os produtos para diagnóstico de doenças infecto-parasitárias (como Aids, Chagas, leishmanioses, etc.) somaram perto de 3 milhões de reações. Investe pesadamente na geração de novas vacinas e kits, bem como no aperfeiçoamento dos existentes. A produção de vacina penta-valente (DTP+HiB+Hepatite B) está em fase de negociação com o Instituto Butantan e a empresa Glaxo-Smith Kleyne.

$\mathrm{Na}$ área de medicamentos, Far-Manguinhos produz oito dos doze anti-retrovirais, além de cerca de 50 outros pro- dutos para doenças negligenciadas e medicamentos de uso contínuo nos programas de saúde pública do país. A produção de medicamentos alcançou cerca de 750 milhões de unidades farmacêuticas em 2001.

$\mathrm{O}$ faturamento da Fiocruz em insumos para o setor saúde ultrapassou R $\$ 250$ milhões em 2001, propiciando um resultado financeiro expressivo, quase totalmente aplicado no financiamento do desenvolvimento.

\section{Serviços de Referência em Saúde}

É da pesquisa em saúde, conforme antes definida, que se origina a capacidade institucional de desenvolver tecnologias em saúde, formar recursos humanos, produzir bens e serviços, apoiar tecnicamente o SUS, de servir como centros de referência assistenciais e diagnósticos, controlar a qualidade de produtos e serviços e de atuar na área da informação, educação e comunicação em saúde.

A Fundação tem firmada, ao longo do tempo, por sua localização no âmbito federal do sistema, a posição de $l i$ derança de redes em saúde no país, mesmo que em certos casos esta função tenha caráter informal. Referem-se às redes formais ou informais identificadas no Diagrama 1: - o conjunto de centros de referência para diagnóstico de doenças infecto-parasitárias e o desenvolvimento de pesquisas e novas metodologias nesta área - localizados no IOC, CPqHEC, CPqAM, CPqGM, CPqRR e ENSP - caracterizam a Fiocruz como liderança e o padrão mais elevado de referência da rede de laboratórios de saúde pública para o sistema de vigilância epidemiológica do país. No caso dos Estados em que está presente (RJ, MG, BA e PE) a Fiocruz vem assumindo também um papel de apoio nos respectivos sistemas estaduais;

- o INCQS, que tem atribuição formal de liderança e referência na Rede Nacional de Laboratórios para a Vigilância Sanitária;

- a ENSP, que faz parte de uma enorme e bem constituída rede de escolas de saúde pública e programas de capacitação na área, desenvolvendo um intenso trabalho de cooperação para este fim com a Associação Brasileira de Pós-Graduação em Saúde Coletiva (Abrasco);

- a Escola Politécnica da Saúde, que tem atribuições formais de Secretaria Técnica da Rede Nacional de Centros Formadores de Nível Médio do país;

- o IFF, que lidera a Rede Nacional de Bancos de Leite Humano e uma rede de centros de neonatologia; 
- outras redes, com as quais a Fiocruz mantém relações ou pode vir a compor, como um sistema nacional de bibliotecas na área da saúde, os museus da área da saúde, uma rede de Escolas de Governo em Saúde, etc.

Esta função de referência e apoio técnico que a Fiocruz desempenha no SUS está quase sempre referida ao grande campo da saúde pública (vigilâncias epidemiológica e sanitária, formação de recursos humanos, etc.), mas também se refere ao componente de saúde individual, por exemplo, por meio da referência e do desenvolvimento de protocolos para a assistência, que fazem o IFF e o CPqHEC.

Essa atividade de referência - que empresta grande visibilidade e legitimidade à Fiocruz - é fortemente dependente de pesquisa \& desenvolvimento que, como foi dito antes, é o cerne da atividade institucional. De fato, a identificação de melhores alternativas e padronizações metodológicas para diagnóstico e controle de qualidade, padrões para bancos de leite humano, estabelecimento de protocolos clínicos baseados em evidências, etc. são parte fundamental da atividade de pesquisa nos centros de referência, que, ao fim, se transformarão em novas práticas no SUS.

Utilizando para a Fiocruz uma analogia com o sistema de saúde dos EUA, poderíamos invocar as atividades referenciais e as recomendações em clínicas e saúde pública do NIH, CDC e FDA - não adotadas compulsoriamente pelos agentes do sistema de saúde, mas com grande poder e influência, por estarem baseadas em sólidas evidências científicas oriundas da pesquisa de qualidade, o que confere enorme legitimidade a elas.

Deve-se destacar, ainda, a função que desempenha a Fiocruz na capacitação de recursos humanos para estes sistemas, seja pela pós-graduação sensu stricto (mestrados e doutorados acadêmicos e, mais recentemente, com os mestrados profissionais), seja na capacitação por diversas modalidades (atualizações, aperfeiçoamentos, especializações, estágios, etc.), presenciais ou a distância, por meio dos quais é difundido o conhecimento referencial. Esta função de capacitação deve ser, portanto, fortemente desenvolvida, pois é das mais eficazes para a afirmação da posição de cooperante com o SUS que deve marcar ainda mais a Fiocruz do futuro.

Ainda de forma analógica, pode-se afirmar que a Fiocruz é, na realidade, o instituto nacional de saúde pública do Brasil, e que corresponde às funções complexas que, em países mais desenvolvidos, cabe a um elenco de organizações. E, como tal, espera-se que com o desenvol- vimento do sistema de saúde do país, cujo vértice é a descentralização, estruturem-se o equivalente a institutos estaduais de saúde ou conjuntos articulados de organizações que, nesse nível do sistema, cumpram este papel. Fica em discussão este tópico e, conseqüentemente, as relações da Fiocruz com tais organizações.

De outro lado, sedia inúmeros centros de referência continentais e mundiais, reconhecidos pelo sistema OMS/OPS.

\section{CONCLUSÕES}

No contexto das extraordinárias mudanças que se verificam globalmente e no país, nos campos social, econômico e da ciência e tecnologia, bem como na vida das organizações, a Fiocruz vem procurando redesenhar seus programas de trabalho e refazer seu modelo de planejamento e gestão, buscando com isso cumprir com maiores eficácia e eficiência sua missão institucional.

A principal orientação estratégica definida em seu Plano Quadrienal 2001-2005, que direciona toda a atividade da Instituição, é o compromisso com a inovação, para melhor responder aos desafios, necessidades e demandas da sociedade nos campos da saúde e da ciência e tecnologia, com seus programas de pesquisa e desenvolvimento, ensino, produção, serviços de referência e informação e comunicação.

Para tanto, definiu e está implementando um modelo de planejamento e gestão participativos, objetivos de longo prazo perfeitamente vinculados às orientações das políticas de saúde e ciência e tecnologia e atuação na fronteira do conhecimento da biologia e da saúde pública.

\section{NOTAS}

1. Para uma análise comparativa das trajetórias da Fiocruz e do Instituto Pasteur, sob a ótica de suas missões e organização, ver Quental e Cabral (2002).

2. Para elucidar melhor este conceito, ver o documento de contribuição da Fiocruz à Conferência Nacional de Ciência, Tecnologia e Inovação (Fiocruz, 2001a).

\section{REFERÊNCIAS BIBLIOGRÁFICAS}

BENCHIMOL, J. Manguinhos, do sonho à vida: a ciência na belle époque. Rio de Janeiro, Fiocruz/COC, 1989.

BUSS, P.M. Discurso de posse como presidente da Fiocruz. Rio de Janeiro, Fundação Oswaldo Cruz, 2001.

DE MASI, D. A emoção e a regra. Grupos criativos na Europa 18501950. São Paulo, José Olympio, 1997. 
FIOCRUZ. Diretrizes para a formulação do plano quadrienal 20012005. Rio de Janeiro, Fundação Oswaldo Cruz, 2001a.

$$
\text { . Conferência nacional de CT\&I: proposições da Fiocruz }
$$
para a área da saúde. Rio de Janeiro, Fundação Oswaldo Cruz, 2001 b, mimeo.

. Programa de desenvolvimento tecnológico em insumos para a saúde (PDTIS). Rio de Janeiro, Fiocruz, 2002a, mimeo.

. Programa de desenvolvimento tecnológico em saúde pública (PDTSP). Rio de Janeiro, Fiocruz, 2002b, mimeo.

. Catálogo de cursos 2002. Rio de Janeiro, Fiocruz, 2002c.

MINISTÉRIO DA CIÊNCIA E TECNOLOGIA. Livro Verde - O debate necessário: Ciência, tecnologia e inovação - Desafio para a sociedade brasileira. Brasília, MCT, 2001.

MINISTÉRIO DA SAÚDE. Agenda da Saúde. Brasília, Ministério da Saúde, 2001, mimeo.
QUENTAL, C. e CABRAL, J.R. Fiocruz e Instituto Pasteur: organizações inseridas em sistemas de inovação distinto. Implicações para o desenvolvimento tecnológico. In: CONGRESSO ABIPTI, 2., 2002, Curitiba. Anais... Curitiba, maio 2002.

SALLES FILHO, S. (coord.). Ciência, tecnologia e inovação. A reorganização da pesquisa no Brasil. Campinas, Ed. Komedi, 2000.

Paulo Marchiori Buss: Médico, Presidente da Fundação Oswaldo Cruz (buss@fiocruz.br).

Paulo Gadelha: Médico, Vice-Presidente da Fundação Oswaldo Cruz (gadelha@presidencia.fiocruz.br). 